\title{
Visions for a Molecular Future
}

\author{
Ruhee $^{1}$, Oshin Sangha ${ }^{2}$, Deep Kamal Kaur Randhawa ${ }^{3}$ \\ M .Tech, Department of Electronics and Communication Engineering, Guru Nanak Dev University, \\ Regional Campus, Jalandhar, India ${ }^{1}$ \\ B. Tech, Department of Electronics and Communication Engineering, Guru Nanak Dev University, \\ Regional Campus, India ${ }^{2}$ \\ Associate Professor, Department of Electronics and Communication Engineering, Guru Nanak Dev University, \\ Regional Campus, Jalandhar, India ${ }^{3}$
}

\begin{abstract}
Zest to sustain Moore's Law paved way for CMOS technology that has evolved from microelectronics to nanoelectronics. Further miniaturization is being limited in silicon based CMOS. Hence, alternative materials are being studied for use in electronic devices. Molecules are a very promising candidate. Molecular Electronics promise not only miniaturization but also property self assembly. Molecular electronics is visualized as the future of electronics. Apart from being the solution to the miniaturization problem, it also promises the possibility to assemble a large number of nanoscale objects to form new devices. Here, we discuss the basics of molecular electronics, its components and some of its applications.
\end{abstract}

Keywords: Molecular Electronics; Molecular Wires; Diodes; Transistors; Switches.

\section{INTRODUCTION}

he The ongoing reduction in the feature size of Si based distinguish between two categories 1) Bulk Molecular technology will run into several economic and physical Systems 2) Single Molecular Systems [2].

limitations in the long term. Therefore the evolution of microelectronics into nanoelectronics and the requirements for the future developments of logic circuits is a very important matter. As silicon is approaching its fundamental limits, it can be said that Silicon Valley will soon have a sibling called "molecular valley"[1]. Molecular electronics can compress the fabrication process into a matter of a few hours, all thanks to self assembly property of the molecules. In this chapter the ultimate miniaturization of logic circuits is done by using single molecules; these molecules are considered as small, typically organic molecules and they have several orders of magnitudes smaller than present feature sizes. The single molecules can act as electronic switches and storage elements [2].

The physical properties of the molecules can vary by their structures and these molecules may be made by chemical synthesis. They can organize themselves on surfaces to regular 2-D patterns as well as 3-D patterns. Therefore in future these molecules are the ideal building blocks for designing the high density electronic devices. In 1974, the idea of using molecules to perform electronic functions was born. Aviram and Patner suggested that a molecule with a donor-spacer-acceptor structure would exhibit a diode like I-V characteristic similar to a traditional semiconductor pn-diode [3]. Molecular electronics is an interdisciplinary field. Because it is used in almost all the fields of science for example in Quantum Chemistry, Organic Chemistry, Inorganic Chemistry, Biology, Material Science, Electrical Engineering and also used in Mesoscopic Physics. The molecular electronics is

\section{NEED FOR MOLECULAR ELECTRONICS}

This question comes to the mind of every researcher, sooner or later and of course, and the answers are always personal. Therefore in case of molecular electronics, from the point of view of basic sciences, molecular electronics offers the possibility to investigate electronic and thermal conduction at the smallest imaginable scale and due to this the physics is completely dominated by quantum mechanical effects [4]. The molecular circuits have very small feature dimensions and have the great variety of mechanical, optical and electrical properties of molecules. The molecular junctions are ideal systems which shed new light into the fundamental electron transfer.

These reasons and many others make molecular electronics a very attractive field of basic research. Moreover, we should never forget that the history of science proves that the exploration of new territories and the subsequent discovery of novel phenomena often lead to unexpected technological applications. Also, from the technological point of view, there is need to investigate the use of molecules which are electronically active elements have variety of applications. If we compare the molecular electronics with the silicon based technology then the molecular electronics have many advantages which are given below [5]

1) Size: The reduce size of small molecules (between 1 and $10 \mathrm{~nm}$ ) could lead to a higher packing density of devices with the subsequent advantages in cost, efficiency, and power dissipation. 


\section{International Journal of Advanced Research in Computer and Communication Engineering}

Vol. 4, Issue 5, May 2015

2) Speed: Although most molecules are poorly be considered as promising candidate for molecular wires conductive, good molecular wires could reduce the transit [7]. The simplest chain for use is wire as polyene. It time of typical transistors, reducing so the time needed for an operation.

consists of alternate sequence of single and double bond, which forms a $\pi$ system. In nanoscale manipulations there 3) Assembly and recognition: One can exploit are enormous developments down to a single atom level, specific intermolecular interactions to form structures by due to which it is easy to investigate the electric current nanoscale self-assembly. Molecular recognition can be through the selected $\pi$ system between the electrodes. The used to modify electronic behavior, providing both switching and sensing capabilities on the single-molecule scale.

4) New functionalities: Special properties of molecules, like the existence of distinct stable geometric structures or isomers, could lead to new electronic functions that are not possible to implement in conventional solid state devices.

At high temperature these molecules have some disadvantages such as at high temperature they show instability. Moreover, the fabrication of reliable molecular junctions requires sometimes controlling matter at an unprecedented level, which can be not only difficult, but also slow and costly. But still the advantages described above are sufficient to motivate the exploration of molecule based electronics.

\section{FUNCTIONS}

In the visionary concept of Molecular electronics early investigations have already demonstrated the strong interdependence of chemical structure and electronic transport properties of integrated molecules. By using other stimuli like voltage, light etc. the molecular junction can also be tunable. By using molecules of appropriate choice the set of bundles can be embedded in a circuit. The functions which are used in molecular electronics are totally based upon the electron transport properties [6]. The molecular wires, molecular diodes, resistors, three terminal devices, switches and storage elements are used as functions in the molecular electronics. The following diagram shows the various components of molecular electronics.

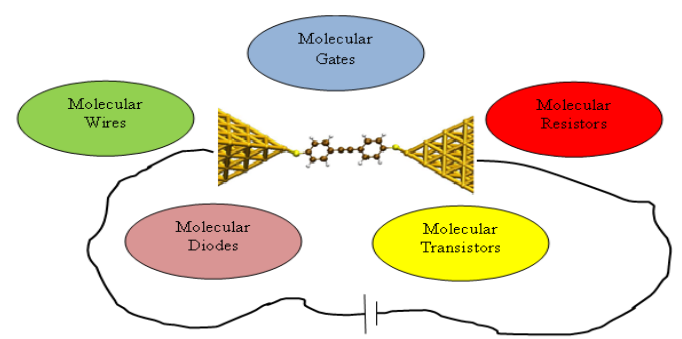

Figure 1: Molecular Electronics

\section{A) MOLECULAR WIRES}

Molecular wires play a very important and basic role in the molecular electronics. A wire is basically a one dimensional object due to which electric charge can transport. They have rod like structures and due to which electrons can transport from one end to another end. It is expected that electron transport will be through the frontier molecular orbitals. Therefore large delocalized $\pi$ systems with reduced HOMO LUMO GAPS (HLG) can single molecules conductance investigations demonstrated substantial differences in electron transport properties between the rods, this emphasize the concept of delocalized $\pi$ systems as molecular wires. The following diagram shows the molecular structure acting as molecular wire [8].

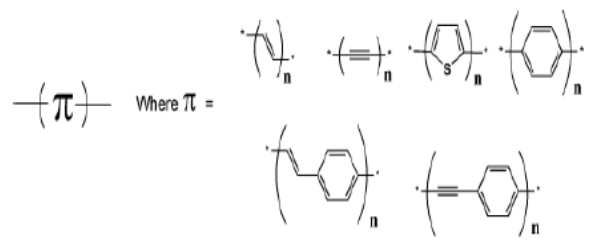

Figure 2: Molecular Structures acting as Molecular wires

\section{B) DIODES}

Molecular rectification was the first hypothetic function described in the field of molecular electronics because the field of molecular electronics is very closely related to the function of diode There are basically two types of diodes 1) Rectifying diode 2) Resonant Tunneling Diode. In both these devices, electrons are driven through one or more potential barriers when the applied voltage exceeds a critical value. The suggested operation of rectifier is based on the principle that there is difference in energy of the frontier molecular orbitals of the 'donor' and 'acceptor'' $\pi$ systems' [9]. The spacer preserves the energy difference between the donor and the acceptor $\pi$ systems, but allows electron transport to certain extent. This behavior of D-6A assembly can be regarded as rectifier function. The acceptor part of the molecule considered as p-type semiconductor while donor part can be regarded as n-type, while the spacer can be compared to the junction barrier. The following fig. 5 shows that the molecular structures which are acting as diodes.
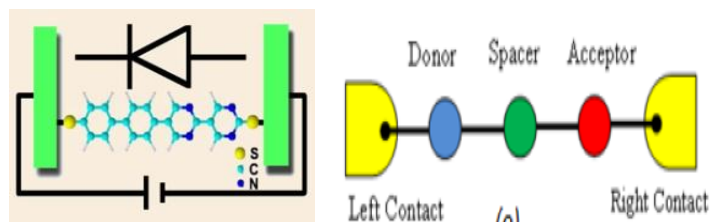

Figure 3: Molecular structures acting as diodes

The two types of molecular diodes are written above they are 1) Molecular Rectifying Diodes 2) Molecular Resonant Tunnelling Diodes. The explanation of these two diodes is given below:

\section{1) Molecular Rectifying diodes}

It is the building block of any three terminal semiconductors for example Bipolar Transistor or a Field Effect Transistor. A diode or a rectifier conducts only in one direction. By using the rectifying diodes at the input 
and connecting a resistor between the supply or the As a result, electrons from the left contact would try to ground, the logic circuit which uses AND/OR gates and flow towards the right contact which is at a higher which are diode based are well known for building logic potential. However, conduction is not possible because the families. According to Aviram and Patner in 1974, a non there is still an energy difference between the Fermi centrosymmetric molecule having appropriate donor and energy of the left contact and the LUMO energy of the acceptor linked with $\sigma-$ bridge and connected with electron donor doped section. It is assumed that both the suitable electrodes will conduct current only in one applied forward and reverse bias potentials are identical. direction and this system was acting like rectifier.

For a higher reverse bias, however, it is possible for the Fermi energy of the left contact to come in resonance with The lowest unoccupied molecular orbital (LUMO) and the the LUMO energy of the electron donor doped section highest occupied molecular orbital (HOMO) can be causing a large current to flow in reverse direction and this aligned in such a way that the conduction can be possible is akin to the breakdown condition in a diode. The above only in one direction [11]. The proposed structure and mono-molecular diode can be used as a building block for schematic representation of the monomolecular diode is realizing molecular digital logic circuits. shown;

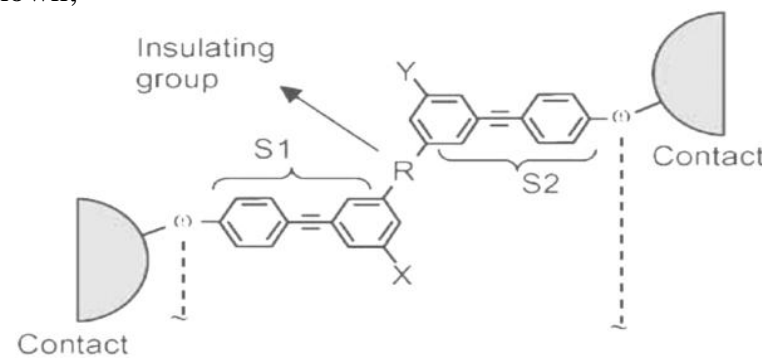

Figure 4: Structure of molecular diode integrally Embedded in a poly-phenylene based molecular conducting wire.

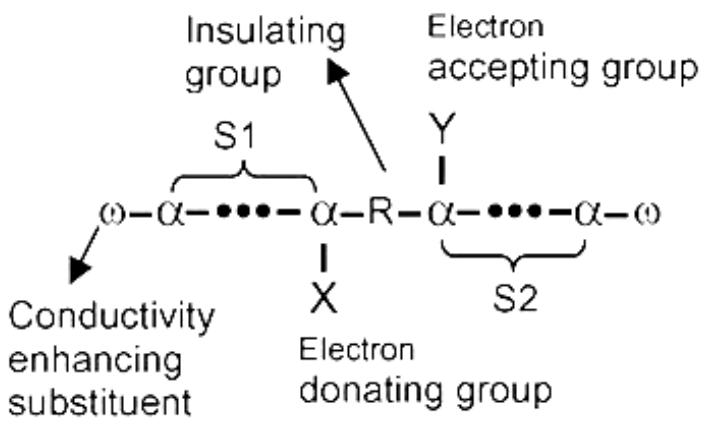

Figure 5: Schematic representation of the molecular diode.

In molecular rectifying diode there are three potential barriers that provide the required isolation between the various parts of the structure. The electrons are induced to flow by tunneling through the three potential barriers from right to left and causing a forward current flow from left to right. The band diagram under the reverse bias condition is shown below in fig. 8 .

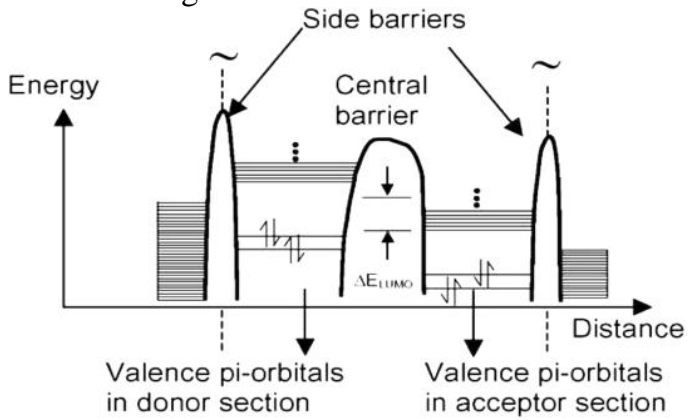

Figure 6: Orbital energy diagram of the rectifying diode under reverse bias condition.
2) Molecular Resonant Tunneling Diode

The devices which have resonant tunnelling structure are said to be resonant tunnelling devices. The RTDs employ quantum effects in their simplest form. In RTD, the electrons can tunnel through some resonant states at certain energy levels. The simplest resonant tunnelling devices are resonant tunnelling diodes and resonant Tunnelling transistors RTDs can be fabricated from layers of two different III-V semiconductor alloys called as binary semiconductors like GaAs and AlAs[13].

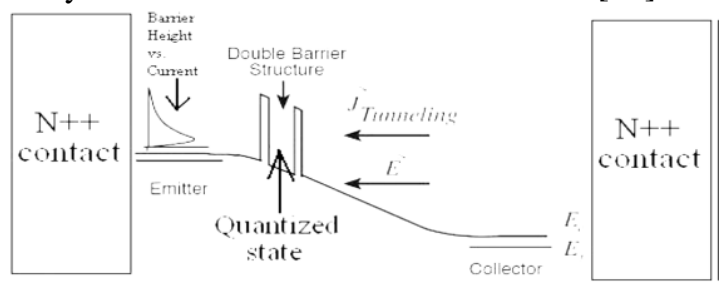

Figure 7: Structure of resonant tunnelling diode.

To achieve a maximum frequency up to $2.2 \mathrm{THz}$ as opposed to $215 \mathrm{GHz}$ in conventional CMOS (Complementary Metal-oxide Semiconductor Transistors), RTDs are used. RTDs have very high switching speed. Therefore, they can be used in a variety of applications in wide band secure communication systems, higher resolution radar and imaging systems for low visibility environment. The resonant tunnelling diodes have much lower capacitance when compare to the Esaki diode; this allows resonant tunnelling diodes to oscillate faster.

a) Operation of Resonant Tunnelling Diode

The resonant tunnelling diode is made by placing two insulating barriers in a semiconductor to create an island where electrons can exist. The quantum mechanics confined their energies to one finite number of discrete quantized levels, when the electrons are restricted between two closely spaced barriers. For the operation of the resonant tunneling diode, energy quantization is the basis.

The tunnelling diode consists of a PN junction in which both $\mathrm{p}$ and $\mathrm{n}$ regions are degenerately doped. There are empty states in the valence band of the $p$ type material and high concentration of electrons in the conduction band of the $\mathrm{n}$ type. Initially, the diode is in thermal equilibrium with no external bias voltage. Therefore, the Fermi level is 
constant. When the forward bias voltage applied the Fermi sized single electron transistor could function at room level start to decrease in $\mathrm{p}$ type and increase in $\mathrm{n}$ type. At temperature. the time when the depletion region is very narrow, the electrons can tunnel through easily and create a forward current as shown in the fig. 10.

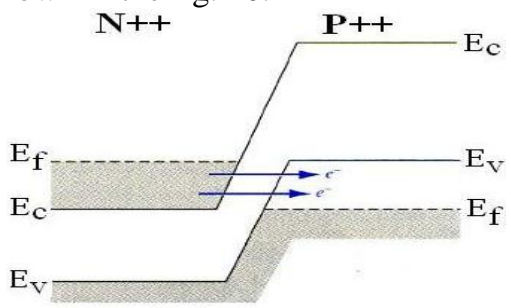

Figure 8: Band diagram showing maximum current across diode.

As the bias voltage continues to increase the ideal diffusion current will cause the current to increase. After that when the reverse bias voltage is applied, the electrons in the $\mathrm{p}$ region are aligned with empty states in the $\mathrm{n}$ region causing a large reverse bias tunnelling current[13].

\section{B) Three Terminal Devices}

Three terminal devices are integral element of an electronic circuit as they provide power amplification to the signal. But the main problem in realization of three terminal devices is structuring three independent nanometer sized leads. Transistor type devices are important for build up integrated circuits (ICs) to perform logic operations because they are also able to provide power amplification to the signal. Three terminal devices are of two types 1) Molecular Transistor 2) molecular Single Electron Transistor. The explanation of these two types is given below.

\section{1) Molecular Transistor}

Molecular transistor has a molecule inserted between two metallic or semiconducting terminals. The third electrode gate is electro statically coupled to the molecule. The gate in a molecular transistor determines the conductance between the source and the drain electrode by controlling the density of charge carriers between them. The basic structure of molecular transistor is shown below:

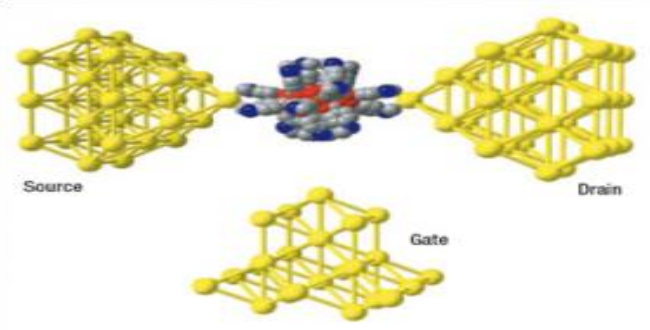

Figure 9: Basic Structure of Molecular Transistor

\section{1) Molecular Single Electron Transistor}

Due to the miniaturization of size in switching devices, the law of quantum mechanics cannot stay forever. Therefore, in order to solve this problem for semiconductor field, the single electron devices were developed. These artificially structured single electron transistors were studied to operate at low temperatures. But the molecular or atomic

\section{a) Structure and Working}

Due to small size, SETs have low power dissipation. Even at high speed the SET have low power dissipation. SET consist of two tunnel junctions sharing one common electrode with low self capacitance; that is known as island [6]. The electrical potential of island can be tuned by a third electrode (the gate) capacitively coupled to the island. In blocking state, the energy is not accessible with tunnelling range of electron.

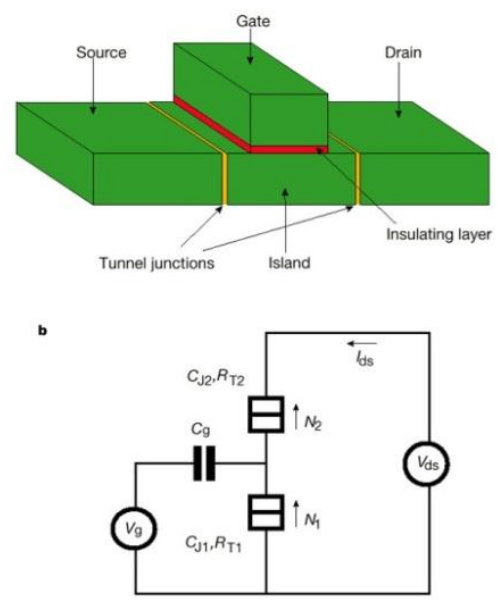

Figure 10: Simplified structure of SET

When a positive voltage is applied to the gate electrode, the energy levels of island electrode are lowered. The electron can tunnel onto the island. From there it can tunnel onto the drain electrode Fermi level. All the energy levels of island electrode are spaced by the separation of $\Delta \mathrm{E}$. Basically, $\Delta \mathrm{E}$ is the energy needed for each subsequential electron to island and to act as a self capacitance ' $C$ '.

As the value of capacitance decreases, the value of $\Delta \mathrm{E}$ increases. In other words, the capacitance and $\Delta \mathrm{E}$ are inversely proportional to each other in a SET. Single electron devices are categorized as: 1) Coulomb Blockade device. It is a SET device with three terminals based on coulomb blockade principle. 2) Nano flash memory based devices. It also has three terminals but it is without tunnel barrier between source and drain. 3) Yano type based devices. It is a two terminal device; information is stored in deep traps in poly-Si.

C) Switches and Storage Elements

Switches allow the control of current flow and these are the basic control element in electronic architecture. Switches can be connected in arrays of multiple switches and can also be used in isolated form to implement logic operations. The property of certain molecules to exist in two different states having different conductive properties leads to the idea for using these molecules as storage molecules. The two stable states of the molecule can be used to depict logic states 0 and 1 . Rotaxanes and catenanes have been synthesize to switch as a function of applied potential between two states. 


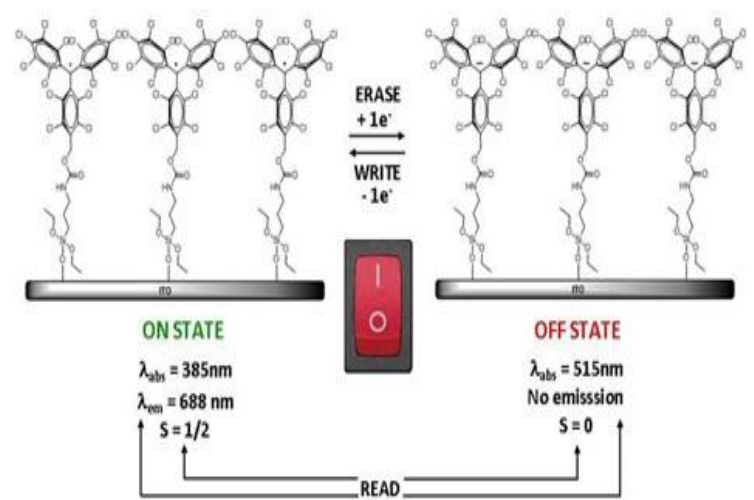

Figure 11: Molecular structures acting as molecular switches and storage elements.

\section{CONCLUSION}

In conclusion, with the use of molecules in electronics the ongoing miniaturization in electronic circuits can be continuing. The molecular electronics concept shows very good potential for the long term future of information technology. Molecular electronics is very faster, smaller, more efficient and potentially more flexible in multiple ways than conventional electronics. Moreover in this field the ultimate density of logic and memory functions, very low power consumption, low fabrication cost makes a very great promise to molecular electronics.

\section{REFERENCES}

[1]. J.M. Tour , (2000), Acc. Chem. ,Vol. 33, pp. 791

[2]. A. Aviram and M. R. Ratner , (1974), Chem. Phys. Lett. , Vol. 29, pp. 277

[3]. A. Aviram and M. R. Ratner , (1998), Acad of sci. , pp. 852

[4]. P. Packan , (1999), Science, Vol. 24, pp. 2079

[5]. James R. Heath and Mark A. Ratner , (2003), "Molecular Electronics", Physics Today, pp. 43-44.

[6]. M. A. Reed and J. M. Tour, (2000), Scientific American, Vol. 282. Pp. 86

[7]. W. B. Davis, W. A. Svec, M. A. Ratner, (1998), Nature, Vol. 60 , pp. 398

[8]. J.M. Tour, (1996), Chem. Rev., Vol. 573, pp. 96

[9]. J. Reichert, R. Ochs, D. Beckmann,(2002), Phys Rev. Lett., Vol. 88, pp. 176804

[10]. M. Jagdesh Kumar, (2007), "Molecular Diodes and Applications", Recent patents on Nanotechnology, Vol. 1, pp. 51

[11]. Geddes NJ et al. ,(1990), "Fabrication and investigation of asymmetric current-voltage characteristics of a metal langmuirblodgett monolayer metal Structure" Appl Phys Lett ; Vol. 56, pp. 19

[12]. Geddes Nt et al. ,(1992)." The electrical properties of metalsandwiched langmuir-blodgett multilayer's and monolayers of a redox-active organic molecular compound" Appl Phys; Vol. 71, Issue.2, pp. 756-768.

[13]. Ruhee, Oshin Sangha, Dr. Deep Kamal Randhwa, Nanoelectronics- An Effort to Sustain Moore's Law, International Journal of Advance Research In Science And Engineering, IJARSE, Vol. No.4, Special Issue (01), March 2015.

\section{BIOGRAPHIES}

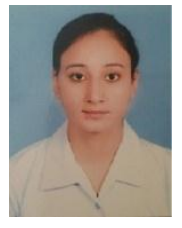

Ruhee, pursuing M.Tech in the field of Electronics and Communication. Her thesis is the study of the variation in the electrical conductivity of carbon nano tubes. Her publications include review papers on Carbon Nanotubes published in a National

Conference, Review on Fullerenes, published in an International Conference. Review on Nanoelectronics, published in the International journal IJARSE and poster published by International Nano Science Community.

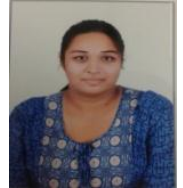

Oshin Sangha, currently pursuing B.Tech in the field of Electronics and Communication. Her publications include review paper on Carbon Nanotubes published in a National Conference, Review on Nanoelectronics, published in the International journal IJARSE and poster published by International Nano Science Community.

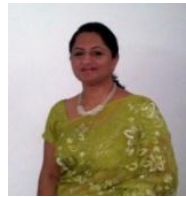

Deep Kamal Kaur Randhawa, currently working as an Assistant Professor at Guru Nanak Dev University, Regional Campus, Jalandhar, Punjab, India. Her field of research and interest is Nanoelectronics. Her publications include review and research papers published at both national and international level journals and conferences. 